\title{
IGF2BP3 Promotes Lung Cancer Progression Through FTO Dependent m6A Modification by Stabilizing N-myc
}

\section{Xiaolin Wang}

Northern Jiangsu People's Hospital and Clinical Medical College of Yangzhou University

\section{Yong Chen}

Northern Jiangsu People's Hospital and Clinical Medical College of Yanzhou University

\section{Lingfeng Min}

Northern Jiangsu People's Hospital and Clinical Medical College of Yangzhou University

Hongcan Shi

Northern Jiangsu People's Hospital and Clinical Medical College of Yangzhou University

\section{Shichun Lu}

Northern Jiangsu People's Hospital and Clinical Medical College of Yangzhou University

Chao Sun

Northern Jiangsu People's Hospital and Clinical Medical College of Yangzhou University

Weiguo Jin

Northern Jiangsu People's Hospital and Clinical Medical College of Yangzhou University

Yusheng Shu ( $\square$ shuyusheng11@163.com )

Northern Jiangsu People's Hospital and Clinical Medical College of Yangzhou University

\section{Research}

Keywords: Lung cancer, IGF2BP3,N6-methyladenosine (m6A), FTO, N-myc

Posted Date: December 29th, 2020

DOI: https://doi.org/10.21203/rs.3.rs-135593/v1

License: (c) (1) This work is licensed under a Creative Commons Attribution 4.0 International License. Read Full License 


\section{Abstract}

Background: N6-methyladenosine modification has been involved in various biological processes. However, its role in non-small cell lung cancer has not been well studied. Here, we show that IGF2BP3, as an transcription factor, plays a critical oncogenic role in non-small-cell lung cancer carcinogenesis through activating FTO expression and inducing aberrant m6A modification.

Methods: To evaluate the role of IGF2BP3 in non-small-cell lung cancer, we performed cell proliferation and cell cycle assays in three lung cancer cell lines. Lung cancer mouse model is used to examine the effects of IGF2BP3/FTO/N-myc on lung proliferation potentials in vivo. We analyzed the correlation between IGF2BP3 and FTO, IGF2BP3 and N-myc protein in colon cancer patients by Pearson correlation. To finally explore the relationship of IGF2BP3/FTO/N-myc, we used western blots, proliferation and cell cycle assays to confirm that IGF2BP3 may regulate lung cancer progression through FTO dependent m6A modification by stabilizing $\mathrm{N}$-myc.

Results: We first identified that IGF2BP3 overexpressed in non-small-cell lung cancer tissue and cells. Then, we showed that FTO was the dysregulated factor responsible for the abnormal N6methyladenosine modification in non-small-cell lung cancer. The loss-of-function assay demonstrated that IGF2BP3 enhances FTO-mediated cell proliferation and promotes cell apoptosis, through regulating expression of target gene $\mathrm{N}$-myc by reducing $\mathrm{m} 6 \mathrm{~A}$ level in mRNA transcript.

Conclusion: Our study demonstrates the functional importance of IGF2BP3 and N6-methyladenosine methylation modification in the tumor progression of non-small-cell lung cancer, and provides profound insights into lung carcinogenesis and drug response.

\section{Background}

Lung cancer is one of the most common malignant tumors worldwide, accounting for $22 \%$ of cancerrelated mortality in China [1]. Non-small-cell lung cancers (NSCLC) constitutes approximately $80 \%$ of lung cancer [2]. Thus, improvements in the diagnostics and therapies of NSCLC are urgently needed. During the past decades, emerging new targeted medicines and therapies have remarkably improved the outcome of NSCLC $[3,4,5]$. While there were still many patients remains poor prognosis due to metastasis when diagnosed. Therefore, it is of great importance to understand the molecular mechanism underlying lung cancer initiation and progression.

The insulin-like growth factor-2 messenger RNA-binding protein 3 (IGF2BP3) is a type of messenger RNA (mRNA) binding proteins, which regulate the translation of insulin-like growth factor-2 (IGF2) [6, 7]. Recently, intensive studies reported that IGF2BP3 dysregulated in many types of cancers $[8,9]$. However, the mechanism by which IGF2BP3 is regulated in human lung cancer is not fully understood.

Emerging studies have showed that N6-methyladenosine (m6A) modification plays a critical role in differentiation, mRNA splicing, cell metabolism and DNA damage response $[10,11,12,13]$. In mammals, 
m6A is modified by the m6A methyltransferases METTL3 and METTL14, erased by a-ketoglutaratedependent dioxygenase ALKB homolog 5 (ALKBH5) or fat-mass and obesity-associated protein (FTO), and read by YTH N6-Methyladenosine RNA Binding Proteins (YTHDFs) or IGF2BPs $[14,15,16]$. The dysfunction of the m6A process was observed in various types of tumorigenesis. In gastric cancer, METTL3-mediated m6A modification is critical for epithelial-mesenchymal transition and metastasis [10]. Suppression of m6A reader YTHDF2 promotes hematopoietic stem cell expansion by regulating the stability of multiple mRNAs critical for HSC self-renewal [17]. Despite these recent discoveries between m6A modification with malignant cancer development and treatment, the status of m6A modification and the underlying regulatory mechanism in lung cancer remains little known.

$\mathrm{N}$-myc, a member of the Myc family of basic-helix-loop-helix-zipper (bHLHZ) transcription factors, is an oncogene associated with a range of cellular processes, including growth,proliferation, apoptosisand differentiation [18]. N-Myc induce malignance by binding to specific DNA sequences and modulating target gene transcription, leading to cell proliferation [19]. Accumulating evidence demonstrates that $\mathrm{N}$ myc exerts diverse biological functions in cancer development [20,21, 22], however, the impact of $\mathrm{N}$-myc in NSCLC development and progression has yet to be investigated.

Our previous study has indicated that TRIM11 acts as an oncogene in lung cancer through promoting cell growth, migration and invasion[23]. In the current study, we investigated the role of m6A modification and IGF2BP3 in lung cancer and addressed the underlying mechanisms by which m6A participates in the biology of lung cancer. Initially, by data mining of the TCGA database, we found that the expression of IGF2BP3 increased in lung cancer compared to corresponding normal tissues and higher IGF2BP3 predicted a poor prognosis in lung cancer patients. Then by loss and gain-of-function assays, we found that IGF2BP3 facilitated cell proliferation and accelerated cell cycle by regulating downstream gene FTO. Finally, m6A-seq combined with RNA-seq analysis revealed that IGF2BP3 may regulate N-myc stability via modulating the $3^{\prime} U T R$ and $5^{\prime} U T R$ region of its transcript.

\section{Materials And Methods}

\section{Bioinformatics analysis}

The gene expression data were obtained from the TCGA dataset (LIHC) for lung adenocarcinoma, including 526 cases of tumor tissues and 59 cases of normal lung tissues.For RNA sequencing, each sample was cleaned up on aRNeasy Mini Column (Qiagen, Limburg, Netherlands), treated with DNase, and analyzed for quality on an Agilent 2100 Bioanalyzer. Samples were on an IlluminaHiSeq 4000 for $2 \times$ 150-bp paired-end sequencing. The sequenced reads were aligned to the human genome GRCh38 by HISAT2 [44]. FeatureCounts [45] was used to quantitate the transcriptome with genome annotation GENCODE v22. Differential analyses were performed to the count files using DESeq2 packages, following standard normalization procedures [46]. The RNA sequence data have been deposited in NCBIs Gene Expression Omnibus (GEO, http://www.ncbi.nlm.nih.gov/geo/) and are accessible through GEO Series accession number GSE150594. For m6A sequencing, samples were sequenced by IlluminaHiSeq 4000 
with single-end 150-bp read length. All reads were mapped to human genome by tophat v2.0.11 with default settings. The m6A level changes for IGF2BP3 knockdown and control cells were calculated by using exomePeak [47].

\section{Clinical specimen collection}

We have collected 35 paired of fresh lung cancer tissues and corresponding paracancerous tissues from patients who underwent surgery in Northern Jiangsu People's Hospital and Clinical Medical College of Yangzhou University from 2015.1 to 2015.12. No the patients had received radiotherapy or chemotherapy before surgery. All patients participating in the study signed written informed consent.

\section{Cell line and treatment}

Lung cancer cell lines PC9, H1975 and H385 were purchased from ATCC (American type culture collection). All lung cancer cells were cultured in DMEM (PC9; SH30243.01, Hyclone) or RPMI-1640 ( $\mathrm{H} 1975$ and $\mathrm{H} 385$; SH30809.01B, Hyclone) medium with $10 \% \mathrm{FBS}$ (fetal bovine serum) at $37^{\circ} \mathrm{C}$ with $5 \%$ $\mathrm{CO}_{2}$. The siRNAs and plasmids were transfected into cell lines using DharmaFECT 1 transfection reagent (Thermo Scientific, USA) or Lipofectamine 3000 (Invitrogen, USA) according to the manufacturer's instruction. All the siRNAs were synthesized and purchased from Genepharm Technologies (China). The siRNA and plasmids sequences involved in the study were listed Supplementary Table 1.

\section{Lentivirus construction}

Vector and cell line used in the study

\begin{tabular}{|ll|}
\hline Name & Company \\
\hline PLKO.1 (Lentivirus core plasmid) & Addgen \\
\hline pLVX-Puro (Lentivirus core plasmid) & Clontech \\
\hline psPAX2, pMD2G (Lentiviral packaging plasmid) & Addgen \\
\hline Competent cell DH5a & Transgen \\
\hline 293T cells (for packaging viruses) & ATCC \\
\hline
\end{tabular}

The RNAi sequence and CDS region sequence specific for the gene (IGF2BP3, FTO, N-myc) which containing the restriction site (upstream EcoR I, downstream BamH I) were synthesized. The synthetic double-strand siRNA annealing was ligated into the pLKO.1-puro vector to form the pLKO.1-puro-shRNA construct, and the CDS sequence was ligated into the PLVX-Puro vector, followed by transformation and plasmid extraction. Lentiviral packaging was performed by lipofection (LipofectamineTM 2000, Invitrogen) in 293T cells. 
RNA in tissues or cells was extracted with Trizol reagent (1596-026, Invitrogen), then transcribed into cDNA using a reverse transcription kit (\#K1622, Fermentas). The prepared CDNA was subjected to the reaction procedure at $95^{\circ} \mathrm{C}$ for $10 \mathrm{~min} \otimes\left(95^{\circ} \mathrm{C}, 15 \mathrm{Sec} ; 60^{\circ} \mathrm{C}, 45 \mathrm{Sec}\right) \times 40$ using a SYBR Green PCR Kit (\#K0223, Thermo) on a Real-time detector (ABI-7300, ABI) for PCR amplification, and the data was analyzed by the software: ABI Prism 7300 SDS Software.

\section{Western blot}

Proteins in tissues or cells were extracted by RIPA buffer (containing protease and phosphatase inhibitor; Solarbio, R0010) and quantified by BCA Quantitation Kit (Thermo, PICPI23223). About $25 \mu \mathrm{g}$ of protein was isolated by $10 \%$ SDS-PAGE (Shanghai JRDUN Biotechnology) and then transferred to a polyvinylidene fluoride (PVDF) membrane (Millipore, HATF00010) by electro-blotting. The cells were blocked for $1 \mathrm{~h}$ in $5 \%$ skim milk (BD Biosciences, BYL40422), and incubated for $2 \mathrm{~h}$ at room temperature. The cells were washed 5 times with PBST and incubated with secondary antibody (1:1000, Beyotime) at $37^{\circ} \mathrm{C}$ for $1 \mathrm{~h}$ in the dark. The chemiluminescence detection reagent (Millipore, WBKLS0100) was added and incubated for $5 \mathrm{~min}$ in the dark and then exposed on the ECL (Tanon, Tanon-5200). Relative protein levels were calculated using Image $\mathrm{J}$ software.

\section{Cell proliferation}

Cells in the logarithmic growth phase were trypsinized and counted under a microscope to prepare a cell suspension of $3 \times 10^{4} \mathrm{cells} / \mathrm{ml}$. Control and transfected NSCLC cells were planted into 96-well culture plates and cultured at $37^{\circ} \mathrm{C}$ overnight. Cell Counting Kit-8 was added to the cells at $0,24,48$, and $72 \mathrm{~h}$. After incubating for $2 \mathrm{~h}$ for $37^{\circ} \mathrm{C}$ in a $5 \% \mathrm{CO}_{2}$ incubator, the absorbance at $450 \mathrm{~nm}$ wavelength was measured with a microplate reader.

\section{Cell cycle}

Cells in logarithmic growth phase were trypsinized and inserted into a six-well plate at a density of 300,000 cells/well. NSCLC cells were seeded into 6-well plates and transfected with siRNA or plasmid. 48 $\mathrm{h}$ after transfection, cells were harvested and fixed with pre-chilled $70 \%$ ethanol at $-20^{\circ} \mathrm{C}$ overnight. After centrifugation, the supernatant was discarded and the cells were washed twice with pre-chilled PBSthe next day. Cells were then stained with $500 \mu \mathrm{L} \mathrm{PI/RNase} \mathrm{staining} \mathrm{buffer} \mathrm{in} \mathrm{the} \mathrm{dark} \mathrm{for} 15$ min. Finally, samples were analyzed using a fluorescence activated cell sorting flow cytometer.

\section{Immunofluorescence}

The pre-treated lung cancer cells were seeded in eight-well chamber slides. Cells were incubated with the corresponding antibodies for $1 \mathrm{~h}$ at room temperature and incubated with fluorescent secondary antibody IgG for half an hour. After DAPI staining and slides mounting, images were captured using a laserscanning confocal microscope. 
Lung cancer cells were transfected with the combinations of N-myc 3'UTR, IGF2BP3 siRNA or control siRNA, and phRL (Renilla luciferase) TK plasmid with Lipofectamine 2000 (Invitrogen, USA). After 12-24 $h$, the luciferase activity was measured using the dual luciferase reporter gene assay system (Promega, USA). Luciferase activity was measured by a luminometer.

\section{RNA Immunoprecipitation}

RNA immunoprecipitation (RIP) analysis was performed using the Magna RIP kit (Millipore). Cells were lysed in RIP lysis buffer and the RNA-protein complexes were immuno-precipitated by using anti-IGF2BP3, anti-YTHDF2 antibodies, and normal rabbit IgG. The co-precipitated RNA was purified using phenol: chloroform: isoamyl alcohol and analyzed by real-time PCR. Prior to immunoprecipitation, the input RNA was subjected to control amplification.

\section{RNA stability assay}

NSCLC cells were treated with Actinomycin D for $0 \mathrm{~h}, 3 \mathrm{~h}$ and $6 \mathrm{~h}$. After total RNA was extracted from treated cells, Real-time PCR was performed to obtain the relative levels of N-myc mRNA. In short, the degradation rate of mRNA (Kdecay) is calculated by the following formula: In (C / CO) =-Kdecayt. $t$ is the time of transcription suppression and $\mathrm{C}$ is the mRNA level at time t. C0 is the mRNA level at 0 hours in the equation, that is, the mRNA level before the decay begins. Therefore, the half-life (t1 / 2) of the mRNA can be calculated by the following equation: In (1/2) =-Kdecayt1 / 2 .

\section{In vivo xenograft model formation}

Mouse experiments were performed in concordance with the NIH Guidelines. To investigate thefunction of IGF2BP3 on tumor growth in vivo, the male BALB/c nude mice at 5-week-old were used in our study. PC9 $\left(1 \times 10^{6}\right)$ cells were injected subcutaneously into these mice to form a lung cancer xenograft model. Then the mice were randomly divided into four groups when the tumor diameter reached $4 \mathrm{~mm}$, and mice were injected with Control shRNA, IGF2BP3shRNA, FTO, and FTO with IGF2BP3shRNA for 20 days by multi-point intratumoral injection. Tumor volume $\left(\mathrm{mm}^{3}\right)$ is estimated by the following formula: tumor volume $\left(\mathrm{mm}^{3}\right)=$ shorter diameter ${ }^{2} \times$ longer diameter $/ 2$.

\section{Statistical analysis}

All the statistical analyses in our study were carried out using SPSS 20.0 software and GraphPad Prism 7. Results were presented as means \pm SEM. Survival analysis was evaluated by the Kaplan-Meier survival curve and Log-rank tests. Statistical significance was assessed by unpaired two-tailed Student's test. $p<$ 0.05 was considered to be statistically significant.

\section{Results}

Upregulated-IGF2BP3 is clinical relevant in NSCLC First, we discovered that the expression of IGF2BP3 was significantly increased in lung cancer samples in the TCGA database, compared with normal tissues 
(Figure 1A). And the survival rate of the patients with high IGF2BP3 expression was significantly lower than that with low expression (Figure 1B). Moreover, we detected IGF2BP3 expression in 35 paired lung cancer and adjacent tissues by using qPCR technology in cohort 1 , and found that the expression of IGF2BP3 was significantly higher in lung carcinoma than the adjacent tissue (Figure 1C). Besides, we detected IGF2BP3 protein level in cohort 2 by using IHC technology and the survival analysis revealed that patients with high IGF2BP3 expression had a poor prognosis (Figure 1D). In addition, we found that high IGF2BP3 expression was positively corelated with lung cancer poor differentiation and lymph node metastasis (Table 1). Moreover, IGF2BP3 was significantly elevated in multiple lung cancer cell lines A549, H1975, H358, H1299, and PC9, which compared with bronchial epithelial HBE cells (Supplementary Figure 1A-1B). All those data indicated that IGF2BP3 may play a crucial role in lung cancer progression, and its high expression may be associated with poor prognosis of lung cancer. IGF2BP3 positively regulate FTO expression To evaluate the specific mechanism of IGF2BP3 involved in lung cancer. We next detected the expression of demethylase (FTO, ALKBH5) and methyltransferase (METTL 3 and METTL 14) in lung cancer cells transfected with IGF2BP3 siRNA or control siRNA. It was found that FTO expression significantly decreased in IGF2BP3 downregulated cells (Figure 2A, Supplementary Figure 2A-2B). We further detect the expression of FTO in lung cancer tissues. The results showed that FTO significantly increased in lung cancer, and was positively correlated with the expression of IGF2BP3 (Figure 2B-2C). Down-regulation of IGF2BP3 expression in lung cancer cell lines PC9 and H1975 resulted in decreased expression of FTO in mRNA and protein level (Figure 2D-2E). Meanwhile, overexpression of IGF2BP3 notably increased FTO expression (Figure 2F, Supplementary Figure 2C). It demonstrated that FTO may be downstream of IGF2BP3. IGF2BP3-induced cell survival depends on FTO in NSCLC cell We next hypothesized that FTO mediated the biological function of IGF2BP3 in lung carcinoma, we transfected IGF2BP3 siRNA alone and in combination with FTO overexpression plasmids into PC9 and H1975 cell lines (Supplementary Figure 2D,2E). It was found that down-regulation of IGF2BP3 inhibited cell proliferation, arrested cell cycle and decreased the expression of FTO (Figure 3A-3B, 3D-3E, 3G-3H). FTO overexpression significantly rescued IGF2BP3 down-regulation induced decrease in cell proliferation and cell cycle arrest (Figure 3A-3B, 3D-3E, 3G-3H). To further confirm the results, we transfected IGF2BP3 overexpression plasmid in $\mathrm{H} 358$ cell line, it was found that upregulation of IGF2BP3 promoted cell proliferation, cell cycle and increased the expression of FTO (Figure 3C, 3F, 3I). While knockdown of FTO significantly blocked IGF2BP3-induced increase in lung cancer cell proliferation and cell cycle acceleration (Figure 3C, 3F, 3I, Supplementary Figure 2F). These data suggested that IGF2BP3-regulated cell proliferation depends on FTO in lung cancer. To address the role of IGF2BP3 in tumorigenic potential in vivo, we injected PC9 cells into nude mice to constructed a subcutaneous xenograft mouse model. The results showed that knockdown of IGF2BP3 dramatically reduced tumor weight (Figure 4A-4B) and tumor volume (Figure $4 C$ ), and increased the survival rate in nude mice (Figure 4D). However, overexpression of FTO in IGF2BP3 siRNA transfected cells significantly rescued IGF2BP3 down-regulation induced decrease in tumor volume and the survival rate (Figure 4A-4D). In support of the pro-tumor role of IGF2BP3, Ki67 staining revealed that the downregulation of IGF2BP3 decreased tumor cell proliferation in vivo, which could be relieved by FTO overexpression (Figure 4E). Furthermore, we found that downregulation of IGF2BP3 decreased FTO expression in vivo both in mRNA and protein level (Figure 4F). These data 
strongly indicated that IGF2BP3 modulate lung cancer tumor growth by regulating the expression of FTO. Transcriptome-wide m6A-seq and RNA-seq assays to identify potential targets of IGF2BP3 in NSCLC To identify the potential mRNA targets of IGF2BP3 whose m6A levels were increased upon IGF2BP3 downregulation. We conducted transcriptome-wide m6A-sequencing (m6A-seq) (Supplementary Table 2) and RNA-seq (Supplementary Table 3) assay in PC9 cell transfected with IGF2BP3 or control siRNA, separately. The results showed that the downregulation of IGF2BP3 notably increased the total m6A level (Figure 5A). Consisted of the previous researches, the most common m6A motif 'GGAC' was significantly enriched in the m6A peaks (Figure 5B). Furthermore, most of the FTO-binding sites were enriched in CDS region and $3^{\prime} U T R$ (Figure $5 C$ ). We next compared the genes with altered-m6a modifications and mRNA expression between IGF2BP3 siRNA and control siRNA group. The analysis of m6A-seq and RNA-seq revealed a significantly increased m6A methylation and reduced mRNA level in the transcription level of $\mathrm{N}$-myc after knockdown of IGF2BP3 in PC9 cells (Figure 5D-5E). The real-time PCR further confirmed that the expression of N-myc was significantly downregulated in IGF2BP3 knockdown cells (Figure 5F). In addition, the MeRIP-qPCR analyses showed the m6A levels of $\mathrm{N}$-myc was dramatically improved in IGF2BP3 downregulated cells (Figure 5G). To further address the effect of m6A modification on N-myc expression, we constructed N-myc luciferase reporter plasmid, which contained the $3^{\prime} U T R$ or $5^{\prime} U T R$ of Nmyc in the m6A sites. As expected, compared with control siRNA, IGF2BP3 siRNA transfection significantly increased the luciferase activity (Figure $5 \mathrm{H}$ ). To confirm the effect of m6A increase on the stability of N-myc, we conducted RNA stability assay and the results showed that knowndown of IGF2BP3 reduced the half-life of N-myc mRNA in PC9 cells (Figure 5I). We further investigated the role FTO in the regulation of $\mathrm{N}$-myc. The results demonstrated that, compared with control group, FTO downregulation notably decreased N-myc expression (Figure $5 \mathrm{~J}$ ), the luciferase activity of $5^{\prime} / 3^{\prime}$ UTR of Nmyc (Figure 5K-5L) and reduced the half-life of N-myc mRNA (Figure 5M), in accordance with our previous results. As reported, YTHDF2 is an m6A reader responsible for mRNA decay[24]. Downregulation of YTHDF2 significantly improved the mRNA level of N-myc (Figure 5N, Supplementary Figure 2G). RIP assay indicated that YTHDF2 might directly bind to the $3^{\prime} U T R$ and 5'UTR and of N-myc (Figure 50-5P). Furthermore, the half-life of N-myc mRNA was notably decreased in NSCLC cells transfected with YTHDF2 siRNA (Figure 5Q). Altogether, our data demonstrated that IGF2BP3/FTO-mediated m6A modification increased N-myc expression through YTHDF2-dependent mRNA stability. N-myc is functionally important target gene of IGF2BP3 in NSCLC N-myc is a pivotal oncogene in carcinogenesis $[25,26]$. Previous studies have demonstrated that $\mathrm{N}$-myc is remarkably increased in various malignant tumors, as well as in lung cancer $[27,28]$. Here we find that $\mathrm{N}$-myc is significantly upregulated in lung cancer tissue, and the expression of N-myc was positively correlated with IGF2BP3 in our cohort (Figure 6A-6B). N-myc expression was significantly decreased in PC9 and H1975 cells transfected with IGF2BP3 siRNA compared with the control group both in mRNA and protein level (Figure 6C-6D). Ectopic expression of IGF2BP3 improved N-myc mRNA and protein levels (Figure 6E). To further investigate the function of N-myc in IGF2BP3 mediated carcinogenesis in lung cancer. We co-transfected N-myc plasmid with IGF2BP3 siRNA in PC9 and H1975 cells (Supplementary Figure 2H, 2I). The results showed that Nmyc overexpression promoted cell proliferation and rescued IGF2BP3 downregulation induced decrease in cell proliferation and cell cycle arrest in PC9 and H1975 cells (Figure 7A-7B, 7D-7E, 7G-7H). 
Downregulation of N-myc declined lung cancer cell proliferation and blocked IGF2BP3-induced increase in lung cancer cell proliferation and cell cycle acceleration (Figure 7C, 7F, 7L, Supplementary Figure 2J). Taken together, these data indicated that N-myc involved in IGF2BP3 mediated pulmonic carcinogenesis.

\section{Discussion}

\section{Upregulated-IGF2BP3 is clinical relevant in NSCLC}

First, we discovered that the expression of IGF2BP3 was significantly increased in lung cancer samples in the TCGA database, compared with normal tissues (Figure 1A). And the survival rate of the patients with high IGF2BP3 expression was significantly lower than that with low expression (Figure 1B). Moreover, we detected IGF2BP3 expression in 35 paired lung cancer and adjacent tissues by using qPCR technology in cohort 1, and found that the expression of IGF2BP3 was significantly higher in lung carcinoma than the adjacent tissue (Figure 1C). Besides, we detected IGF2BP3 protein level in cohort 2 by using IHC technology and the survival analysis revealed that patients with high IGF2BP3 expression had a poor prognosis (Figure 1D). In addition, we found that high IGF2BP3 expression was positively corelated with lung cancer poor differentiation and lymph node metastasis (Table 1). Moreover, IGF2BP3 was significantly elevated in multiple lung cancer cell lines A549, H1975, H358, H1299, and PC9, which compared with bronchial epithelial HBE cells (Supplementary Figure 1A-1B). All those data indicated that IGF2BP3 may play a crucial role in lung cancer progression, and its high expression may be associated with poor prognosis of lung cancer.

\section{IGF2BP3 positively regulate FTO expression}

To evaluate the specific mechanism of IGF2BP3 involved in lung cancer. We next detected the expression of demethylase (FTO, ALKBH5) and methyltransferase (METTL 3 and METTL 14) in lung cancer cells transfected with IGF2BP3 siRNA or control siRNA. It was found that FTO expression significantly decreased in IGF2BP3 downregulated cells (Figure 2A, Supplementary Figure 2A-2B). We further detect the expression of FTO in lung cancer tissues. The results showed that FTO significantly increased in lung cancer, and was positively correlated with the expression of IGF2BP3 (Figure 2B-2C). Down-regulation of IGF2BP3 expression in lung cancer cell lines PC9 and H1975 resulted in decreased expression of FTO in mRNA and protein level (Figure 2D-2E). Meanwhile, overexpression of IGF2BP3 notably increased FTO expression (Figure 2F, Supplementary Figure 2C). It demonstrated that FTO may be downstream of IGF2BP3.

\section{IGF2BP3-induced cell survival depends on FTO in NSCLC cell}

We next hypothesized that FTO mediated the biological function of IGF2BP3 in lung carcinoma, we transfected IGF2BP3 siRNA alone and in combination with FTO overexpression plasmids into PC9 and H1975 cell lines (Supplementary Figure 2D,2E). It was found that down-regulation of IGF2BP3 inhibited cell proliferation, arrested cell cycle and decreased the expression of FTO (Figure 3A-3B, 3D-3E, 3G-3H). FTO overexpression significantly rescued IGF2BP3 down-regulation induced decrease in cell proliferation 
and cell cycle arrest (Figure 3A-3B, 3D-3E, 3G-3H). To further confirm the results, we transfected IGF2BP3 overexpression plasmid in $\mathrm{H} 358$ cell line, it was found that upregulation of IGF2BP3 promoted cell proliferation, cell cycle and increased the expression of FTO (Figure 3C, 3F, 3I). While knockdown of FTO significantly blocked IGF2BP3-induced increase in lung cancer cell proliferation and cell cycle acceleration (Figure 3C, 3F, 3I, Supplementary Figure 2F). These data suggested that IGF2BP3-regulated cell proliferation depends on FTO in lung cancer.

To address the role of IGF2BP3 in tumorigenic potential in vivo, we injected PC9 cells into nude mice to constructed a subcutaneous xenograft mouse model. The results showed that knockdown of IGF2BP3 dramatically reduced tumor weight (Figure 4A-4B) and tumor volume (Figure 4C), and increased the survival rate in nude mice (Figure 4D). However, overexpression of FTO in IGF2BP3 siRNA transfected cells significantly rescued IGF2BP3 down-regulation induced decrease in tumor volume and the survival rate (Figure 4A-4D). In support of the pro-tumor role of IGF2BP3, Ki67 staining revealed that the downregulation of IGF2BP3 decreased tumor cell proliferation in vivo, which could be relieved by FTO overexpression (Figure 4E). Furthermore, we found that downregulation of IGF2BP3 decreased FTO expression in vivo both in mRNA and protein level (Figure 4F). These data strongly indicated that IGF2BP3 modulate lung cancer tumor growth by regulating the expression of FTO.

\section{Transcriptome-wide m6A-seq and RNA-seq assays to identify potential targets of IGF2BP3 in NSCLC}

To identify the potential mRNA targets of IGF2BP3 whose m6A levels were increased upon IGF2BP3 downregulation. We conducted transcriptome-wide m6A-sequencing (m6A-seq) (Supplementary Table 2) and RNA-seq (Supplementary Table 3) assay in PC9 cell transfected with IGF2BP3 or control siRNA, separately. The results showed that the downregulation of IGF2BP3 notably increased the total m6A level (Figure 5A). Consisted of the previous researches, the most common m6A motif 'GGAC' was significantly enriched in the m6A peaks (Figure $5 B$ ). Furthermore, most of the FTO-binding sites were enriched in CDS region and $3^{\prime} U T R$ (Figure $5 C$ ). We next compared the genes with altered-m6a modifications and mRNA expression between IGF2BP3 siRNA and control siRNA group. The analysis of m6A-seq and RNA-seq revealed a significantly increased m6A methylation and reduced mRNA level in the transcription level of $\mathrm{N}$-myc after knockdown of IGF2BP3 in PC9 cells (Figure 5D-5E). The real-time PCR further confirmed that the expression of N-myc was significantly downregulated in IGF2BP3 knockdown cells (Figure 5F). In addition, the MeRIP-qPCR analyses showed the m6A levels of N-myc was dramatically improved in IGF2BP3 downregulated cells (Figure 5G). To further address the effect of m6A modification on N-myc expression, we constructed N-myc luciferase reporter plasmid, which contained the 3'UTR or 5'UTR of Nmyc in the m6A sites. As expected, compared with control siRNA, IGF2BP3 siRNA transfection significantly increased the luciferase activity (Figure $5 \mathrm{H}$ ). To confirm the effect of m6A increase on the stability of N-myc, we conducted RNA stability assay and the results showed that knowndown of IGF2BP3 reduced the half-life of N-myc mRNA in PC9 cells (Figure 5I). We further investigated the role FTO in the regulation of $\mathrm{N}$-myc. The results demonstrated that, compared with control group, FTO downregulation notably decreased $\mathrm{N}$-myc expression (Figure $5 \mathrm{~J}$ ), the luciferase activity of $5^{\prime} / 3^{\prime} \mathrm{UTR}$ of $\mathrm{N}$ myc (Figure 5K-5L) and reduced the half-life of N-myc mRNA (Figure 5M), in accordance with our 
previous results. As reported, YTHDF2 is an m6A reader responsible for mRNA decay[24]. Downregulation of YTHDF2 significantly improved the mRNA level of N-myc (Figure 5N, Supplementary Figure 2G). RIP assay indicated that YTHDF2 might directly bind to the $3^{\prime}$ UTR and 5'UTR and of N-myc (Figure 50-5P). Furthermore, the half-life of N-myc mRNA was notably decreased in NSCLC cells transfected with YTHDF2 siRNA (Figure 5Q). Altogether, our data demonstrated that IGF2BP3/FTO-mediated m6A modification increased N-myc expression through YTHDF2-dependent mRNA stability.

\section{$\mathrm{N}$-myc is functionally important target gene of IGF2BP3 in NSCLC}

$\mathrm{N}$-myc is a pivotal oncogene in carcinogenesis [25,26]. Previous studies have demonstrated that $\mathrm{N}$-myc is remarkably increased in various malignant tumors, as well as in lung cancer $[27,28]$. Here we find that $\mathrm{N}$ myc is significantly upregulated in lung cancer tissue, and the expression of $\mathrm{N}$-myc was positively correlated with IGF2BP3 in our cohort (Figure 6A-6B). N-myc expression was significantly decreased in PC9 and H1975 cells transfected with IGF2BP3 siRNA compared with the control group both in mRNA and protein level (Figure 6C-6D). Ectopic expression of IGF2BP3 improved N-myc mRNA and protein levels (Figure 6E). To further investigate the function of N-myc in IGF2BP3 mediated carcinogenesis in lung cancer. We co-transfected N-myc plasmid with IGF2BP3 siRNA in PC9 and H1975 cells (Supplementary Figure $2 \mathrm{H}, 2 \mathrm{l}$ ). The results showed that $\mathrm{N}$-myc overexpression promoted cell proliferation and rescued IGF2BP3 downregulation induced decrease in cell proliferation and cell cycle arrest in PC9 and $\mathrm{H} 1975$ cells (Figure 7A-7B, 7D-7E, 7G-7H). Downregulation of N-myc declined lung cancer cell proliferation and blocked IGF2BP3-induced increase in lung cancer cell proliferation and cell cycle acceleration (Figure 7C, 7F, 7L, Supplementary Figure 2J). Taken together, these data indicated that $\mathrm{N}$ myc involved in IGF2BP3 mediated pulmonic carcinogenesis.

\section{Conclusion}

Taken together, here we provide compelling in vitro and in vivo evidence demonstrating that the IGF2PB3 plays a critical oncogenic role in cell proliferation and pulmonary carcinogenesis mediated by FTO, through regulating m6A level in mRNA transcripts of its target gene N-myc. Our study highligts the functional importance of the m6A modification maghinery in lung cancer. Given the importance of IGF2BP3/FTO/N-myc axis in carcinogenesis in lung cancer, targeting this pathway may promising therapeutic strategy in the treatment of lung cancer.

\section{Abbreviations}

m6A N6-methyladenosine

NSCLC Non-small-cell lung cancers

IGF2BP3 The insulin-like growth factor-2 messenger RNA-binding protein 3 
mRNA messenger RNA

ALKBH5 ALKB homolog 5

FTO fat-mass and obesity-associated protein

YTHDFs YTH N6-Methyladenosine RNA Binding Proteins

bHLHZ basic-helix-loop-helix-zipper

RIP RNA immunoprecipitation

\section{Declarations}

\section{Acknowledgements}

Not applicable.

\section{Authors' contributions}

XLW and YSS conducted the study design. XLW, YC, LFM, HCS carried out the assays and collected the samples. XLW performed the statistical analysis. SCL, CS contributed to animal work. YC analyzed the clinical data. XLW drafted the manuscript. YSS revised the manuscript. All authors reviewed and approved the final manuscript.

\section{Funding}

The study was supported by Natural Science Foundation of Yangzhou (YZ2016108) and the 13th 5-yearplan of strengthening scientific medical technology in Yangzhou city (ZDRC201812).

\section{Availability of data and materials}

All data generated or analyzed during this study are included in this published article (and its supplementary information files).

\section{Ethics approval and consent to participate}

All patients participating in the study are fully informed and sign an informed consent. The study was approved by Northern Jiangsu People's Hospital and Clinical Medical College of Yangzhou University ethics committee and was carried out in accordance with the principles outlined in the Declaration of Helsinki.

\section{Consent for publication}

Not applicable. 


\section{Competing interests}

The authors declare no conflict of interest in this research manuscript.

\section{Contributor information}

Xiaolin Wang, E-mail: 1240152721@qq.com

Yong Chen, E-mail: chenyong_jsyz@sina.com

Lingfeng Min, E-mail: minlingfeng@163.com

Hongcan Shi, E-mail: shihongcan@yzu.edu.cn

Shichun Lu, E-mail: yzchest@163.com

Chao Sun, E-mail: simon1028@sina.com

Weiguo Jin, E-mail: doctorjin@sina.com

\section{References}

1. Chen W, Zheng R, Baade PD, Zhang S, Zeng H, Bray F, Jemal A, Yu XQ, He J. Cancer statistics in China, 2015. CA Cancer J Clin. 2016;66:115-32.

2. Goulart BH, Martins RG, Lynch TJ. Twenty-two years of phase III trials for patients with advanced non-small-cell lung cancer: sobering results. J Clin Oncol. 2001;19:4089.

3. Adeegbe DO, Liu S, Hattersley MM, Bowden M, Zhou CW, Li S, Vlahos R, Grondine M, Dolgalev I, Ivanova EV, Quinn MM, Gao P, Hammerman PS, Bradner JE, Diehl JA, Rustgi AK, Bass AJ, Tsirigos A, Freeman GJ, Chen H, Wong KK. BET Bromodomain Inhibition Cooperates with PD-1 Blockade to Facilitate Antitumor Response in Kras-Mutant Non-Small Cell Lung Cancer. Cancer Immunol Res. 2018;6:1234-45.

4. Brody R, Zhang Y, Ballas M, Siddiqui MK, Gupta P, Barker C, Midha A, Walker J. PD-L1 expression in advanced NSCLC: Insights into risk stratification and treatment selection from a systematic literature review. Lung Cancer. 2017;112:200-15.

5. Liu YT, Zhang K, Li CC, Hu XS, Jiang J, Hao XZ, Wang Y, Li JL, Xing PY, Yang S, Zhang X, Wang GQ, Cai SL, Shi YK. Depth of Response was Associated with Progression-Free Survival in Patients with Advanced Non-small Cell Lung Cancer treated with EGFR-TKI. J Cancer. 2019;10:5108-13.

6. Liao B, Patel M, Hu Y, Charles S, Herrick DJ, Brewer G. Targeted knockdown of the RNA-binding protein CRD-BP promotes cell proliferation via an insulin-like growth factor II-dependent pathway in human K562 leukemia cells. J Biol Chem. 2004;279:48716-24.

7. Mancarella C, Pasello M, Manara MC, Toracchio L, Sciandra EF, Picci P, Scotlandi K. Insulin-Like Growth Factor 2 mRNA-Binding Protein 3 Influences Sensitivity to Anti-IGF System Agents Through 
the Translational Regulation of IGF1R. Front Endocrinol (Lausanne). 2018;9:178.

8. Liu Y, Yu C, Wu Y, Sun X, Su Q, You C, Xin H. CD44(+) fibroblasts increases breast cancer cell survival and drug resistance via IGF2BP3-CD44-IGF2 signalling. J Cell Mol Med. 2017;21:1979-88.

9. Zhou Y, Huang T, Siu HL, Wong CC, Dong Y, Wu F, Zhang B, Wu WK, Cheng AS, Yu J, To KF, Kang W. IGF2BP3 functions as a potential oncogene and is a crucial target of miR-34a in gastric carcinogenesis. Mol Cancer. 2017;16:77.

10. Yue B, Song C, Yang L, Cui R, Cheng X, Zhang Z, Zhao G. METTL3-mediated N6-methyladenosine modification is critical for epithelial-mesenchymal transition and metastasis of gastric cancer. Mol Cancer. 2019;18:142.

11. Yu J, Shen L, Liu Y, Ming H, Zhu X, Chu M, Lin J. The m6A methyltransferase METTL3 cooperates with demethylase ALKBH5 to regulate osteogenic differentiation through NF-kappaB signaling. Mol Cell Biochem 2019.

12. Cheng Y, Luo H, Izzo F, Pickering BF, Nguyen D, Myers R, Schurer A, Gourkanti S, Bruning JC, Vu LP, Jaffrey SR, Landau DA, Kharas MG. m(6)A RNA Methylation Maintains Hematopoietic Stem Cell Identity and Symmetric Commitment. Cell Rep. 2019;28:1703-16. e1706.

13. Chen J, Wang C, Fei W, Fang X, Hu X. Epitranscriptomic m6A modification in the stem cell field and its effects on cell death and survival. Am J Cancer Res. 2019;9:752-64.

14. Zhou KI, Pan T. An additional class of m(6)A readers. Nat Cell Biol. 2018;20:230-2.

15. Tang C, Klukovich R, Peng H, Wang Z, Yu T, Zhang Y, Zheng H, Klungland A, Yan W. ALKBH5dependent m6A demethylation controls splicing and stability of long 3'-UTR mRNAs in male germ cells. Proc Natl Acad Sci U S A. 2018;115:E325-33.

16. Dominissini D, Moshitch-Moshkovitz S, Schwartz S, Salmon-Divon M, Ungar L, Osenberg S, Cesarkas $\mathrm{K}$, Jacob-Hirsch J, Amariglio N, Kupiec M, Sorek R, Rechavi G. Topology of the human and mouse m6A RNA methylomes revealed by m6A-sEq. Nature. 2012;485:201-6.

17. Li Z, Qian P, Shao W, Shi H, He XC, Gogol M, Yu Z, Wang Y, Qi M, Zhu Y, Perry JM, Zhang K, Tao F, Zhou K, Hu D, Han Y, Zhao C, Alexander R, Xu H, Chen S, Peak A, Hall K, Peterson M, Perera A, Haug JS, Parmely T, Li H, Shen B, Zeitlinger J, He C, Li L. Suppression of m(6)A reader Ythdf2 promotes hematopoietic stem cell expansion. Cell Res. 2018;28:904-17.

18. Henriksson M, Luscher B. Proteins of the Myc network: essential regulators of cell growth and differentiation. Adv Cancer Res. 1996;68:109-82.

19. Eilers M, Eisenman RN. Myc's broad reach. Genes Dev. 2008;22:2755-66.

20. Qing G, Skuli N, Mayes PA, Pawel B, Martinez D, Maris JM, Simon MC. Combinatorial regulation of neuroblastoma tumor progression by N-Myc and hypoxia inducible factor HIF-1alpha. Cancer Res. 2010;70:10351-61.

21. Marshall GM, Liu PY, Gherardi S, Scarlett CJ, Bedalov A, Xu N, Iraci N, Valli E, Ling D, Thomas W, van Bekkum M, Sekyere E, Jankowski K, Trahair T, Mackenzie KL, Haber M, Norris MD, Biankin AV, Perini G, Liu T. SIRT1 promotes N-Myc oncogenesis through a positive feedback loop involving the effects of MKP3 and ERK on N-Myc protein stability. PLoS Genet. 2011;7:e1002135. 
22. Fletcher JI, Gherardi S, Murray J, Burkhart CA, Russell A, Valli E, Smith J, Oberthuer A, Ashton LJ, London WB, Marshall GM, Norris MD, Perini G, Haber M. N-Myc regulates expression of the detoxifying enzyme glutathione transferase GSTP1, a marker of poor outcome in neuroblastoma. Cancer Res. 2012;72:845-53.

23. Wang X, Shi W, Shi H, Lu S, Wang K, Sun C, He J, Jin W, Lv X, Zou H, Shu Y. TRIM11 overexpression promotes proliferation, migration and invasion of lung cancer cells. J Exp Clin Cancer Res. 2016;35:100.

24. Zhang C, Chen Y, Sun B, Wang L, Yang Y, Ma D, Lv J, Heng J, Ding Y, Xue Y, Lu X, Xiao W, Yang YG, Liu F. m(6)A modulates haematopoietic stem and progenitor cell specification. Nature. 2017;549:273-6.

25. Yin Y, Xu L, Chang Y, Zeng T, Chen X, Wang A, Groth J, Foo WC, Liang C, Hu H, Huang J. N-Myc promotes therapeutic resistance development of neuroendocrine prostate cancer by differentially regulating miR-421/ATM pathway. Mol Cancer. 2019;18:11.

26. Olsen RR, Otero JH, Garcia-Lopez J, Wallace K, Finkelstein D, Rehg JE, Yin Z, Wang YD, Freeman KW. MYCN induces neuroblastoma in primary neural crest cells. Oncogene. 2017;36:5075-82.

27. Tong Q, Ouyang S, Chen R, Huang J, Guo L. MYCN-mediated regulation of the HES1 promoter enhances the chemoresistance of small-cell lung cancer by modulating apoptosis. Am J Cancer Res. 2019;9:1938-56.

28. Liu K, Wang S, Liu Y, Gu J, Gu S, Xu Z, Zhang R, Wang Z, Ma H, Chen Y, Ji L. Overexpression of MYCN promotes proliferation of non-small cell lung cancer. Tumour Biol. 2016;37:12855-66.

29. Wang Z, Tong D, Han C, Zhao Z, Wang X, Jiang T, Li Q, Liu S, Chen L, Chen Y, Li A, Huang C. Blockade of miR-3614 maturation by IGF2BP3 increases TRIM25 expression and promotes breast cancer cell proliferation. EBioMedicine. 2019;41:357-69.

30. Xu W, Sheng Y, Guo Y, Huang Z, Huang Y, Wen D, Liu CY, Cui L, Yang Y, Du P. Increased IGF2BP3 expression promotes the aggressive phenotypes of colorectal cancer cells in vitro and vivo. J Cell Physiol. 2019;234:18466-79.

31. Suvasini R, Shruti B, Thota B, Shinde SV, Friedmann-Morvinski D, Nawaz Z, Prasanna KV, Thennarasu K, Hegde AS, Arivazhagan A, Chandramouli BA, Santosh V, Somasundaram K. Insulin growth factor-2 binding protein 3 (IGF2BP3) is a glioblastoma-specific marker that activates phosphatidylinositol 3kinase/mitogen-activated protein kinase (PI3K/MAPK) pathways by modulating IGF-2. J Biol Chem. 2011;286:25882-90.

32. Kao SH, Cheng WC, Wang YT, Wu HT, Yeh HY, Chen YJ, Tsai MH, Wu KJ. Regulation of miRNA Biogenesis and Histone Modification by K63-Polyubiquitinated DDX17 Controls Cancer Stem-like Features. Cancer Res. 2019;79:2549-63.

33. Sun TT, He J, Liang Q, Ren LL, Yan TT, Yu TC, Tang JY, Bao YJ, Hu Y, Lin Y, Sun D, Chen YX, Hong J, Chen H, Zou W, Fang JY. LncRNA GClnc1 Promotes Gastric Carcinogenesis and May Act as a Modular Scaffold of WDR5 and KAT2A Complexes to Specify the Histone Modification Pattern. Cancer Discov. 2016;6:784-801. 
34. Tsuboi M, Kondo K, Soejima S, Kajiura K, Kawakita N, Toba H, Kawakami Y, Yoshida M, Takizawa H, Tangoku A. Chromate exposure induces DNA hypermethylation of the mismatch repair gene MLH1 in lung cancer. Mol Carcinog 2019.

35. Yan TT, Ren LL, Shen CQ, Wang ZH, Yu YN, Liang Q, Tang JY, Chen YX, Sun DF, Zgodzinski W, Majewski M, Radwan P, Kryczek I, Zhong M, Chen J, Liu Q, Zou W, Chen HY, Hong J, Fang JY. miR-508 Defines the Stem-like/Mesenchymal Subtype in Colorectal Cancer. Cancer Res. 2018;78:1751-65.

36. Li Z, Weng H, Su R, Weng X, Zuo Z, Li C, Huang H, Nachtergaele S, Dong L, Hu C, Qin X, Tang L, Wang Y, Hong GM, Huang H, Wang X, Chen P, Gurbuxani S, Arnovitz S, Li Y, Li S, Strong J, Neilly MB, Larson RA, Jiang $X$, Zhang P, Jin J, He C, Chen J. FTO Plays an Oncogenic Role in Acute Myeloid Leukemia as a N(6)-Methyladenosine RNA Demethylase. Cancer Cell. 2017;31:127-41.

37. Ma JZ, Yang F, Zhou CC, Liu F, Yuan JH, Wang F, Wang TT, Xu QG, Zhou WP, Sun SH. METTL14 suppresses the metastatic potential of hepatocellular carcinoma by modulating $\mathrm{N}(6)$ methyladenosine-dependent primary MicroRNA processing. Hepatology. 2017;65:529-43.

38. Bartosovic M, Molares HC, Gregorova P, Hrossova D, Kudla G, Vanacova S. N6-methyladenosine demethylase FTO targets pre-mRNAs and regulates alternative splicing and 3 '-end processing. Nucleic Acids Res. 2017;45:11356-70.

39. Zhao X, Yang Y, Sun BF, Shi Y, Yang X, Xiao W, Hao YJ, Ping XL, Chen YS, Wang WJ, Jin KX, Wang X, Huang CM, Fu Y, Ge XM, Song SH, Jeong HS, Yanagisawa H, Niu Y, Jia GF, Wu W, Tong WM, Okamoto A, He C, Rendtlew Danielsen JM, Wang XJ, Yang YG. FTO-dependent demethylation of N6methyladenosine regulates mRNA splicing and is required for adipogenesis. Cell Res. 2014;24:140319.

40. Dammert MA, Bragelmann J, Olsen RR, Bohm S, Monhasery N, Whitney CP, Chalishazar MD, Tumbrink HL, Guthrie MR, Klein S, Ireland AS, Ryan J, Schmitt A, Marx A, Ozretic L, Castiglione R, Lorenz C, Jachimowicz RD, Wolf E, Thomas RK, Poirier JT, Buttner R, Sen T, Byers LA, Reinhardt HC, Letai A, Oliver TG, Sos ML. MYC paralog-dependent apoptotic priming orchestrates a spectrum of vulnerabilities in small cell lung cancer. Nat Commun. 2019;10:3485.

41. Ries RJ, Zaccara S, Klein P, Olarerin-George A, Namkoong S, Pickering BF, Patil DP, Kwak H, Lee JH, Jaffrey SR. m(6)A enhances the phase separation potential of mRNA. Nature. 2019;571:424-8.

42. Song $H$, Feng $X$, Zhang $H$, Luo Y, Huang J, Lin $M$, Jin J, Ding X, Wu S, Huang H, Yu T, Zhang M, Hong $\mathrm{H}$, Yao S, Zhao Y, Zhang Z. METTL3 and ALKBH5 oppositely regulate $\mathrm{m}(6) \mathrm{A}$ modification of TFEB mRNA, which dictates the fate of hypoxia/reoxygenation-treated cardiomyocytes. Autophagy. 2019;15:1419-37.

43. Wang X, Lu Z, Gomez A, Hon GC, Yue Y, Han D, Fu Y, Parisien M, Dai Q, Jia G, Ren B, Pan T, He C. N6methyladenosine-dependent regulation of messenger RNA stability. Nature. 2014;505:117-20.

44. Pertea M, Kim D, Pertea G, Leek JT, Salzberg SL. Transcript-level expression analysis of RNA-seq experiments with HISAT, StringTie and Ballgown. Nat Protoc. 2016;11:1650-67.

45. Liao Y, Smyth GK, Shi W. featureCounts: an efficient general purpose program for assigning sequence reads to genomic features. Bioinformatics. 2014;30:923-30. 
46. Love MI, Huber W, Anders S. Moderated estimation of fold change and dispersion for RNA-seq data with DESeq2. Genome Biol. 2014;15:550-0.

47. Meng J, Lu Z, Liu H, Zhang L, Zhang S, Chen Y, Rao MK, Huang Y. A protocol for RNA methylation differential analysis with MeRIP-Seq data and exomePeak R/Bioconductor package. Methods. 2014;69:274-81.

\section{Tables}

Table 1. Relationship between IGF2BP3 expression and clinicopathological features of lung cancer

\begin{tabular}{|c|c|c|c|}
\hline & IGF2BP3 & & $P$ value \\
\hline Clinicopathological features & Low $(n=46)$ & High $(n=54)$ & \\
\hline Gender & & & 0.5790 \\
\hline Male $(n=53)$ & 23 & 30 & \\
\hline Female $(n=47)$ & 23 & 24 & \\
\hline Age (years) & & & 0.4222 \\
\hline$£ 60(n=50)$ & 25 & 25 & \\
\hline$>60(n=50)$ & 21 & 29 & \\
\hline Tumor size (cm) & & & 0.5579 \\
\hline$£ 3(n=49)$ & 24 & 25 & \\
\hline$>3(n=51)$ & 22 & 29 & \\
\hline Tumor differentiation & & & $0.0122^{*}$ \\
\hline Well $(n=18)$ & 11 & 7 & \\
\hline Moderate $(n=29)$ & 18 & 11 & \\
\hline Poor $(n=53)$ & 17 & 36 & \\
\hline Lymph node status (stage) & & & $0.0163^{*}$ \\
\hline NO $(n=31)$ & 20 & 11 & \\
\hline$N 1(n=32)$ & 15 & 17 & \\
\hline N2 (n=37) & 11 & 26 & \\
\hline
\end{tabular}

Differences between groups were done by the Chi-square test.

Figures 
A
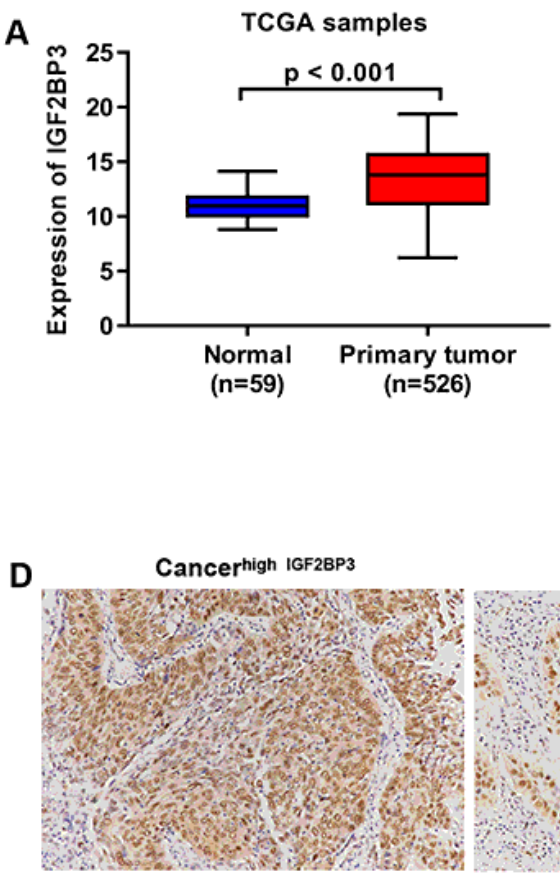

B

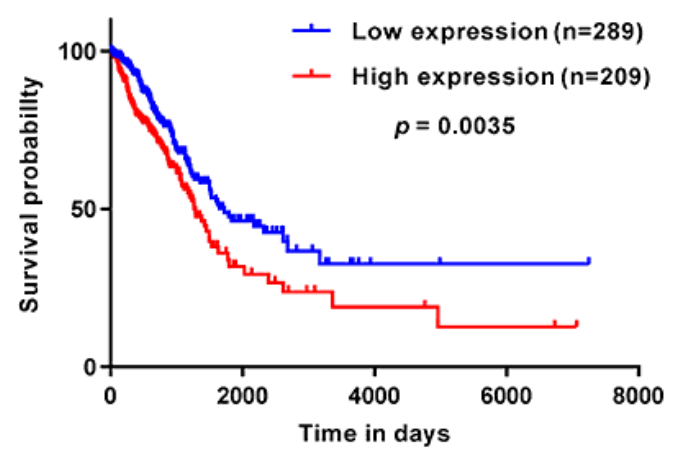

Effect of IGF2BP3 expression level on patient survival

CancerLow IGF2BP3

ParacancerLow IGF2BP3

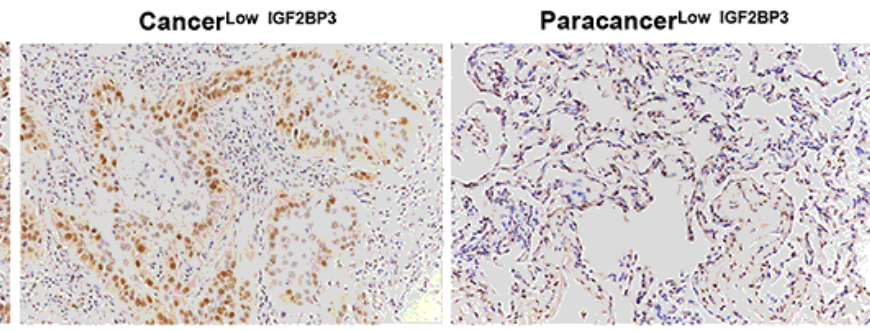

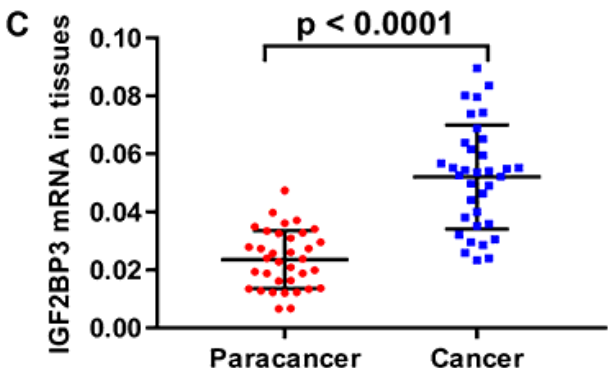

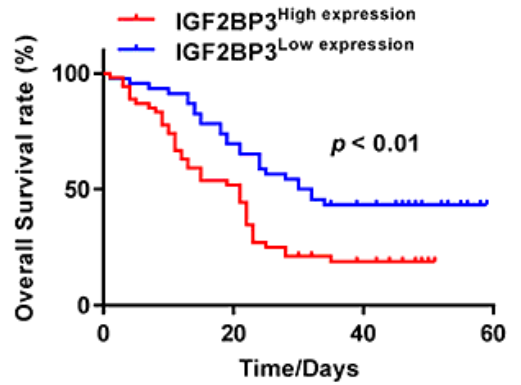

Figure 1

Upregulated-IGF2BP3 is clinical relevant in NSCLC (A) The IGF2BP3 expression in the LUAD database. (B) Kaplan Meier-plotter prognostic analysis between IGF2BP3 low and high group. (C) The detection of IGF2BP3 expression in 35 paired lung cancer and adjacent tissues by Q-PCR. (D) The expression contribution of IGF2BP3 in lung cancer and adjacent tissues by IHC and Kaplan Meier-plotter prognostic analysis between IGF2BP3 low and high group. 
A

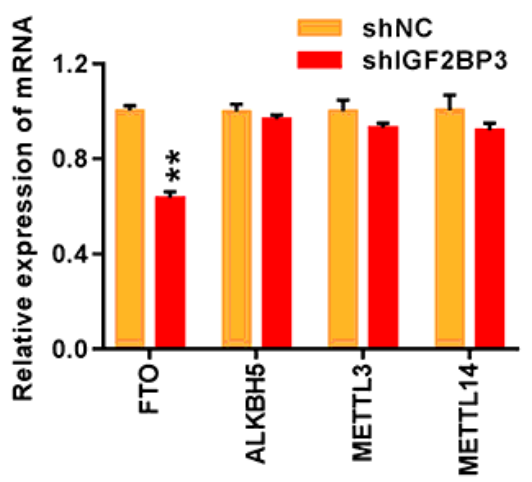

D
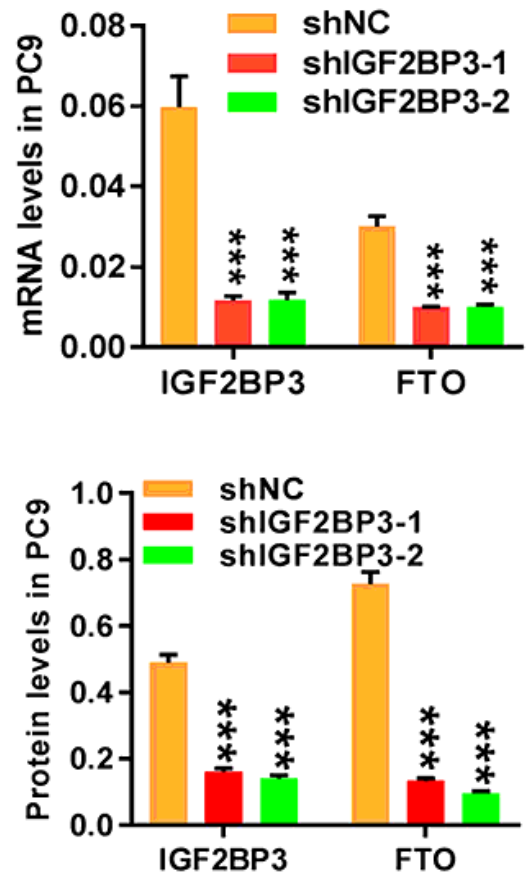

IGF2BP3

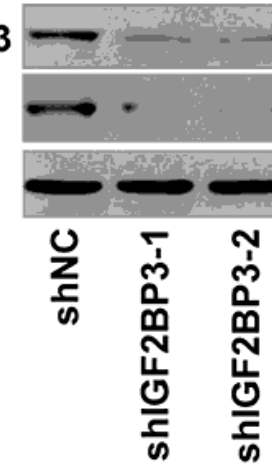

FTO

GAPDH
B

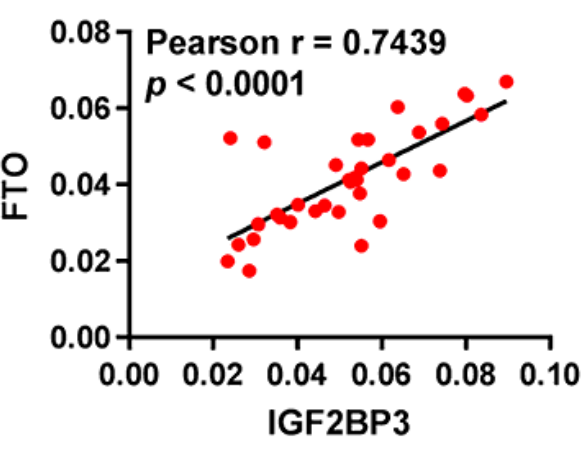

E
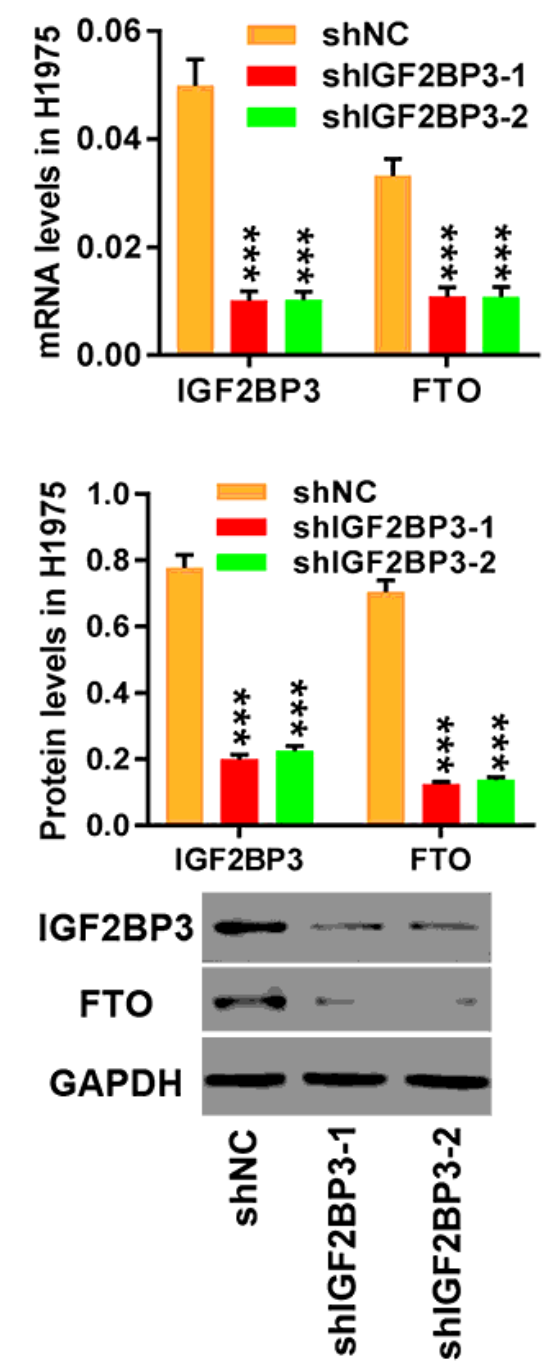

C
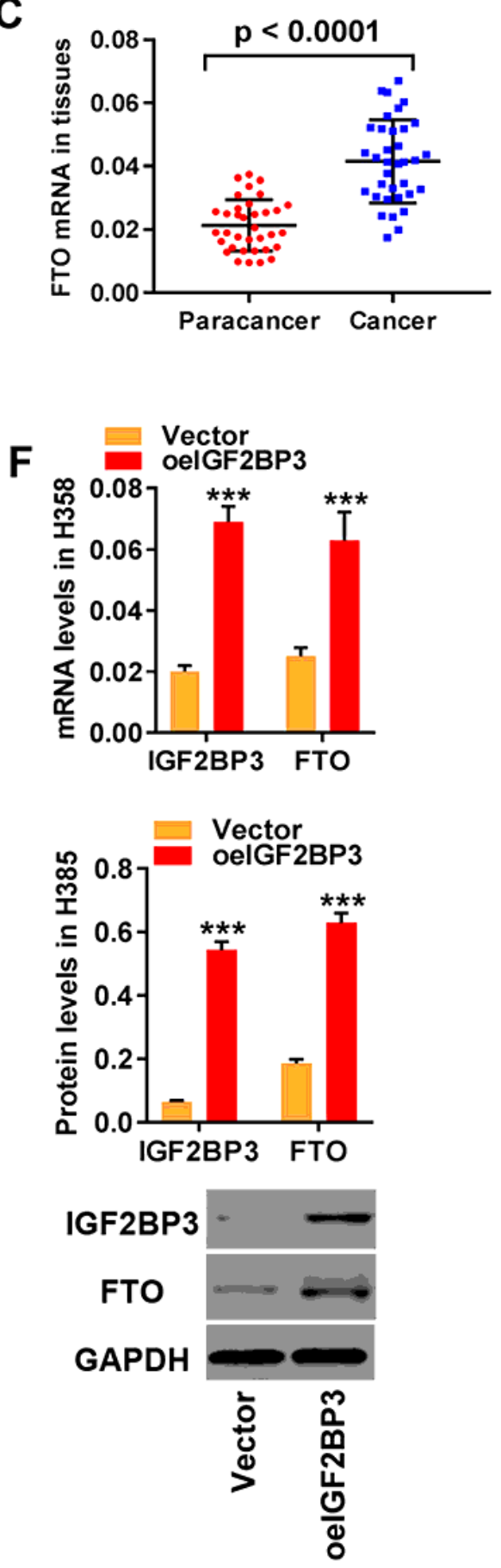

Figure 2

IGF2BP3 positively regulates FTO expression (A) The detection of FTO, ALKBH5, METTL3, and METTL14 expression in the different groups (shNC and shIGF2BP3) by Q-PCR. **p $<0.01$ vs. shNC. (B) The correlation between IGF2BP3 and FTO by Pearson analysis. (C) The detection of FTO expression in 35 paired lung cancer and adjacent tissues by Q-PCR. (D) Q-PCR (top) and WB (bottom) were used to detect the expression of IGF2BP3 and FTO in PC9 cells. (E) Q-PCR (top) and WB (bottom) were used to detect the expression of IGF2BP3 and FTO in H1975 cells. ***p < 0.001 vs. shNC. (F) Q-PCR (top) and WB (bottom) were used to detect the expression of IGF2BP3 and FTO in H358 cells. ***p $<0.001$ vs. Vector. 

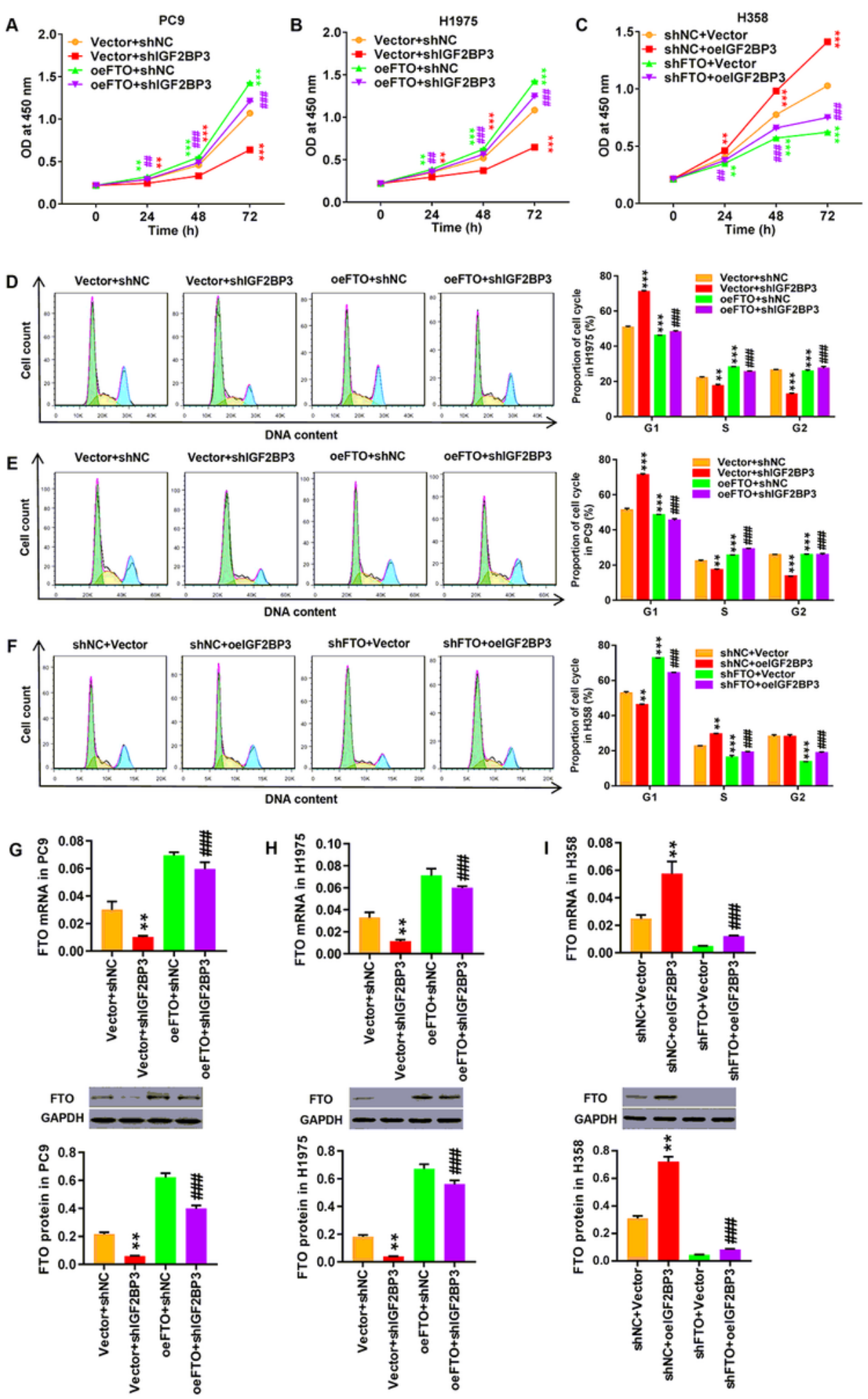

\section{Figure 3}

IGF2BP3-induced cell survival depends on FTO in NSCLC cell (A-C) CCK8 was used to detect cell proliferation in PC9 (A), H1975 (B) and H358 (C) cells in different groups. (D-F) Flow cytometry was used to detect cell cycle state in PC9 (D), H1975 (E) and H358 (F) cells in different groups. (G-H) Q-PCR (top) and WB (bottom) were used to detect the expression of FTO in PC9(G) and H1975 cells (H) after different treatments. (I) Q-PCR (top) and WB (bottom) were used to detect the expression of FTO in H358 cells in 
different groups. ${ }^{\star \star} p<0.01,{ }^{\star \star *} p<0.001$ vs. Vector+shNC or shNC+Vector; \#\#p $<0.01, \# \# \# p<0.001$ vs. Vector+shIGF2BP3 or shNC+oelGF2BP3.
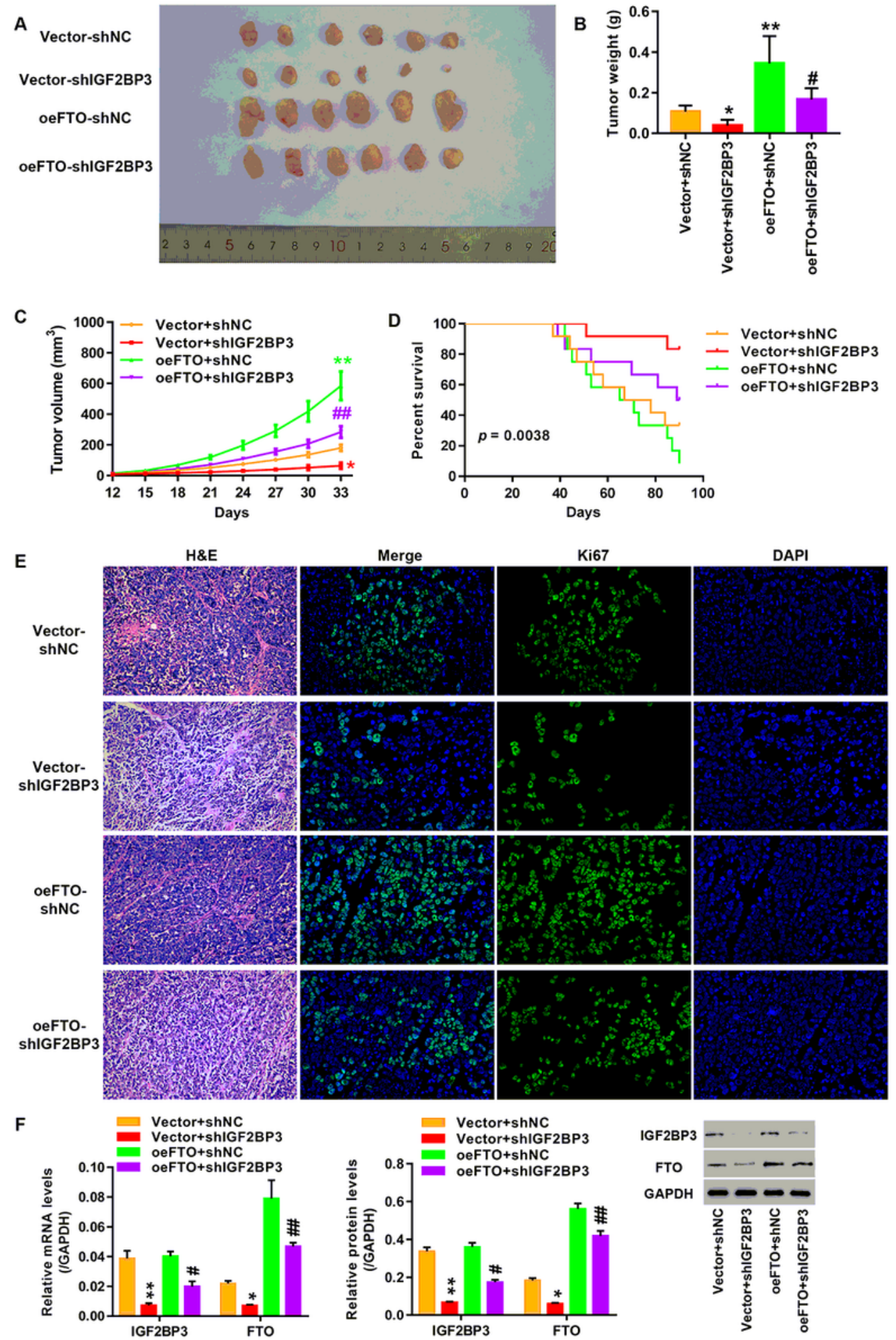

Figure 4

The function of IGF2BP3 in vivo (A) Representative images of tumors.(B-D) Statistical analysis of (B) tumor weights, (C)tumor volumes and (D)survival curve of nude mice in different groups $(n=6$, nonparametric Mann-Whitney test). (E) H\&E and Ki67 staining for tumor cell proliferation analysis. (F) Q- 
PCR and Western blot detection of IGF2BP3, FTO and N-myc expression in different groups. ${ }^{*} p<0.05,{ }^{*} p$ $<0.01$ vs. Vector+shNC; \#p < 0.05, \#\#p < 0.01 vs. Vector+shIGF2BP3.

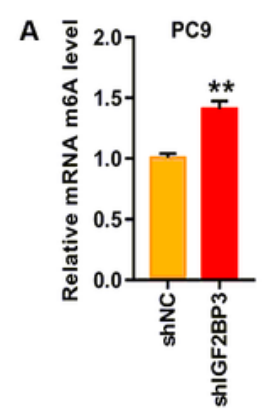

D

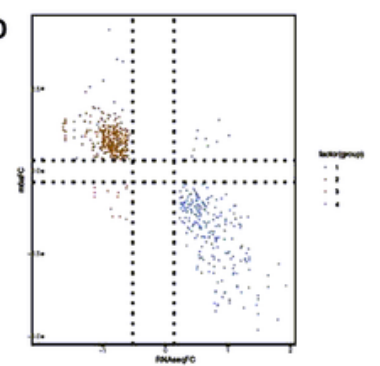

F

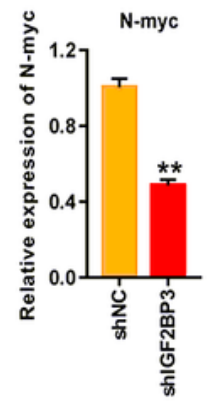

B

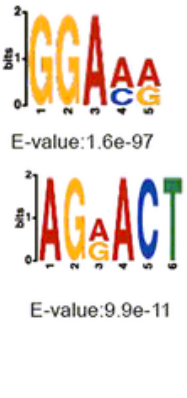

C Distribution of Peaks in Relation to Genes

E

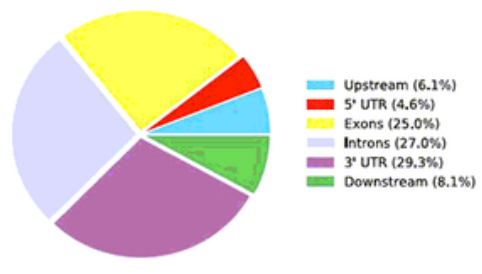

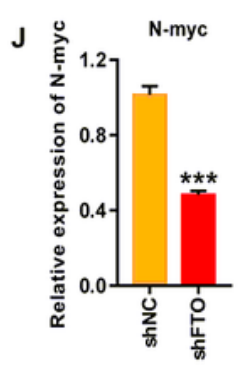

N

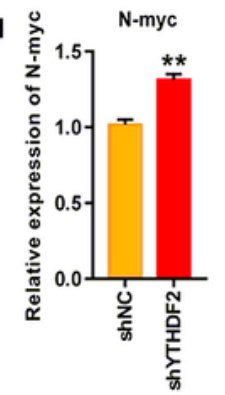

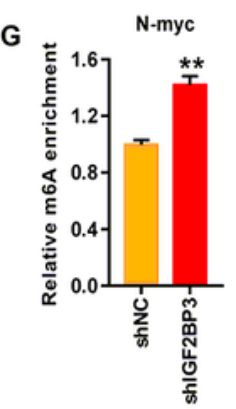
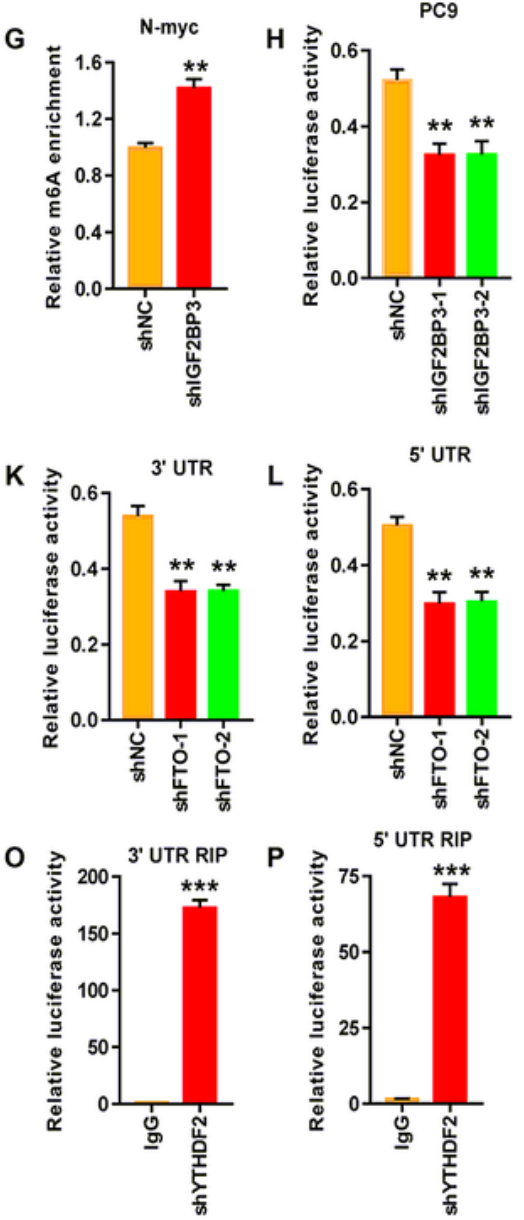

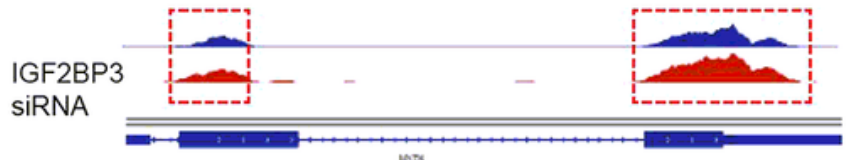

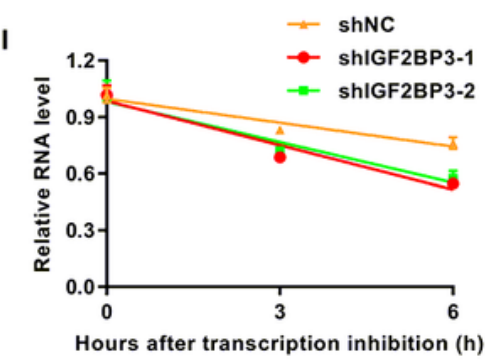
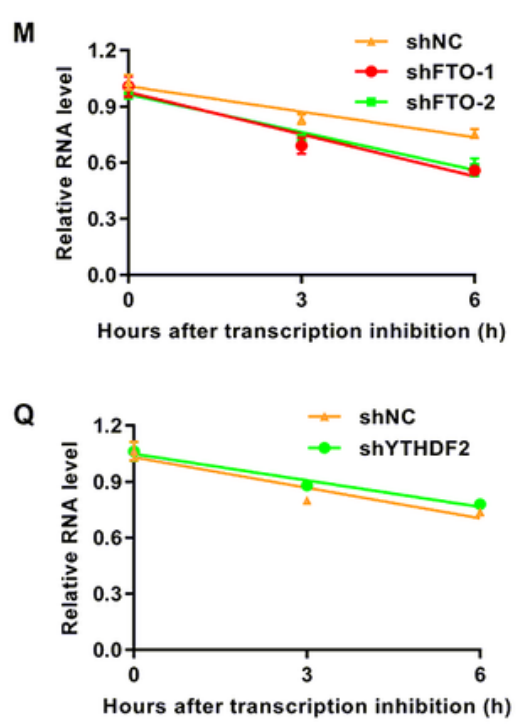

\section{Figure 5}

Transcriptome-wide m6A-seq and RNA-seq assays to identify potential targets of IGF2BP3 in NSCLC (A) The global m6A levels in the mRNA of PC9 cells were measured by ELISA. (B) The motif analysis revealed the top consensus m6A motif in PC9 cells. (C) Graphs of m6A peak distribution illustrating the proportion 
of common m6A peaks in the indicated regions in PC9 cells. (D) Distribution of genes with a significant change in both m6A level and gene expression level in IGF2BP3 siRNA transfected PC9 cells compared with control siRNA cells. (E) The relative abundance of m6A sites along N-myc mRNA in IGF2BP3 siRNA transfected PC9 cells compared with control siRNA cells, as detected by m6A-seq. (F) The detection of Nmyc mRNA in PC9 cells transfected with IGF2BP3-siRNA and control-siRNA by real-time PCR. (G) The detection of m6A enrichment in PC9 cells by the MeRIP-qPCR analysis. $(H)$ Relative luciferase activity of wild-type or mutant N-myc 3'UTR firefly luciferase reporter in PC9 cells treated with IGF2BP3-siRNA and control-siRNA. ${ }^{*} p<0.01$ vs. shNC. (I) The N-myc mRNA half-life (t1/2) in PC9 cells transfected with control-siRNA or IGF2BP3-siRNA. (J) The detection of N-myc mRNA in PC9 cells transfected with FTOsiRNA and control-siRNA by real-time PCR. (K-L) Relative luciferase activity of wild-type or mutant N-myc 3'UTR (K) and 5'UTR (L) firefly luciferase reporter in PC9 cells treated with FTO-siRNA and control-siRNA. ${ }^{\star \star} p<0.01,{ }^{* \star} \mathrm{p}<0.001$ vs. shNC. (M) The N-myc mRNA half-life (t1/2) in PC9 cells transfected with control-siRNA or FTO-siRNA. (N) The detection of N-myc mRNA in PC9 cells transfected with YTHDF2siRNA and control-siRNA by real-time PCR. ${ }^{*} p<<0.01$ vs. shNC. (O-P) RIP-qPCR showed the binding of YTHDF2 to N-myc HK2 3'UTR (O) and 5'UTR (P). ***p < 0.001 vs. IgG. (Q) The N-myc mRNA half-life (t1/2) in PC9 cells transfected with control-siRNA or YTHDF2-siRNA. 

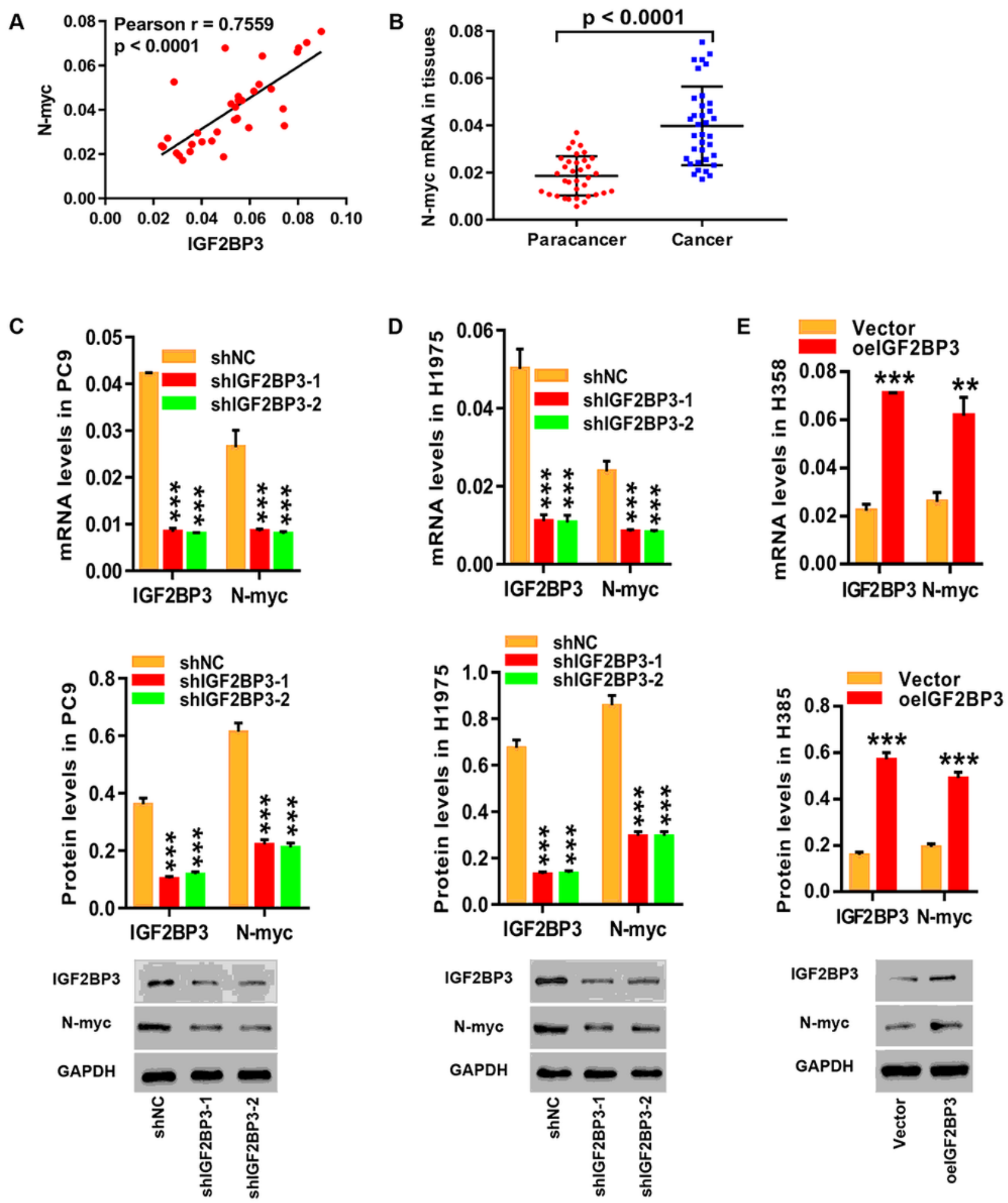

Figure 6

IGF2BP3 positively regulates N-myc expression (A) The correlation between IGF2BP3 and N-myc by Pearson analysis. (B) The detection of $\mathrm{N}$-myc expression in 35 paired lung cancer and adjacent tissues by Q-PCR. (C-D) Q-PCR (top) and WB (bottom) were used to detect the expression of IGF2BP3 and N-myc in PC9 (C) and H1975 (D) cells. ${ }^{* \star}$ p $<0.001$ vs. shNC. (E) Q-PCR (top) and WB (bottom) were used to detect the expression of IGF2BP3 and N-myc in H358 cells. ${ }^{* \star} p<0.01$, ${ }^{* \star *} \mathrm{p}<0.001$ vs. Vector. 

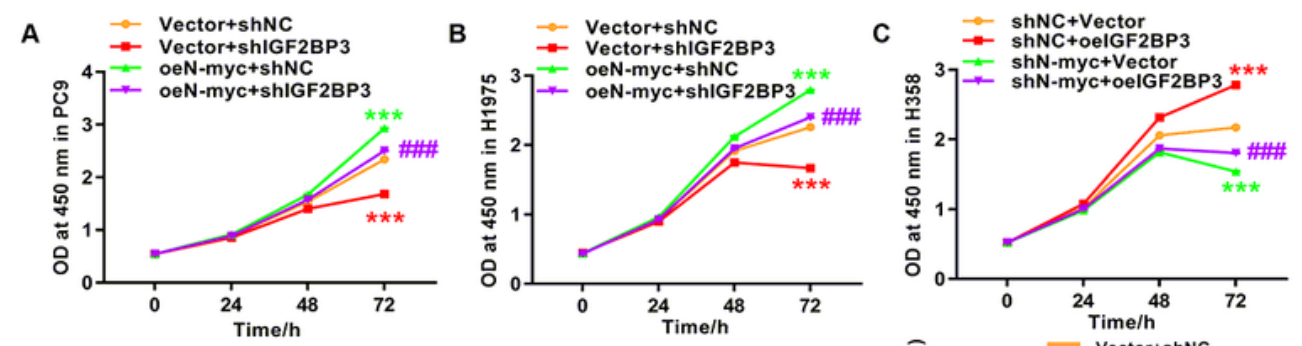

D
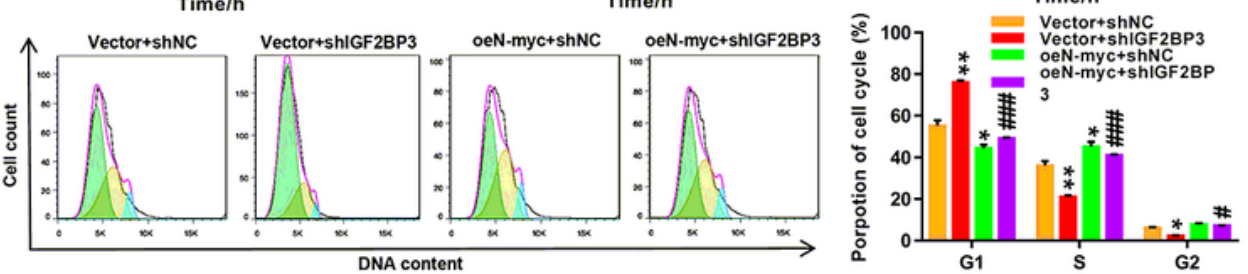

E
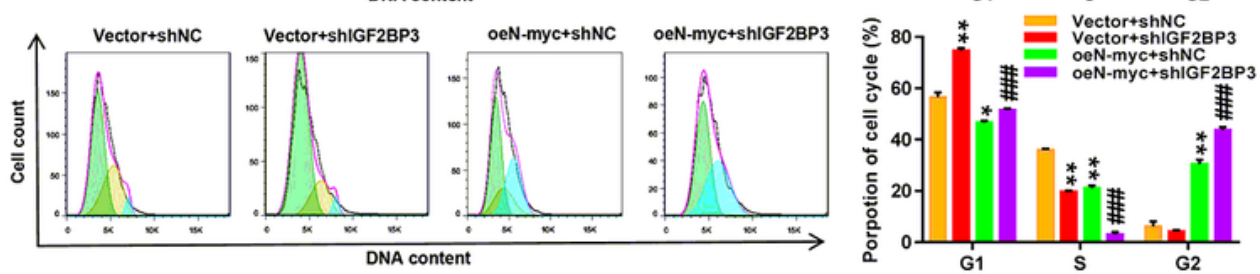

$\mathbf{F}$
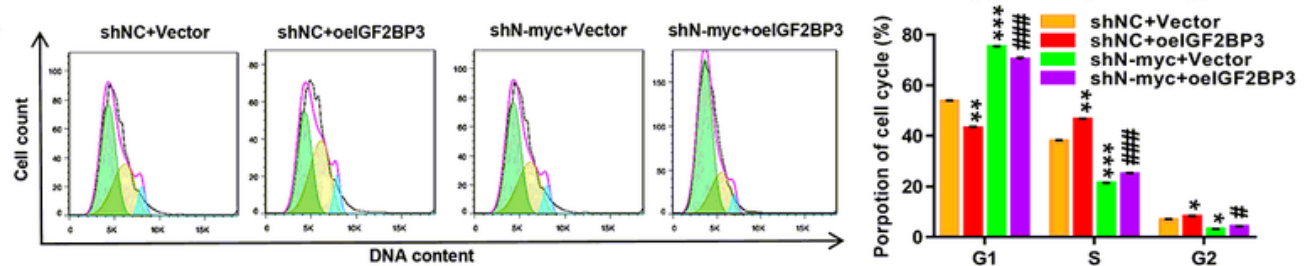

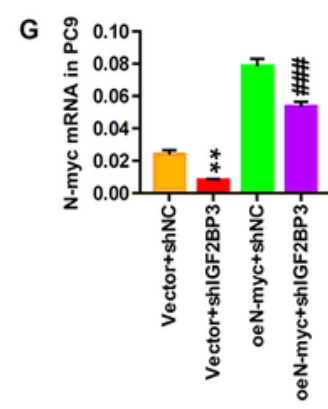

GAPDH $-\cdots-$

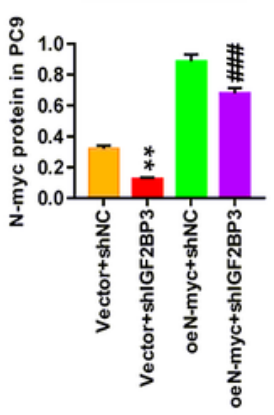

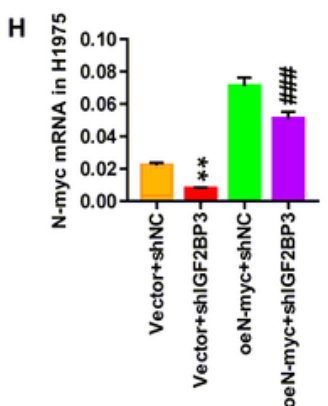

N-myc $=-$

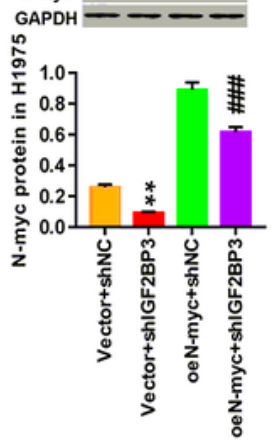

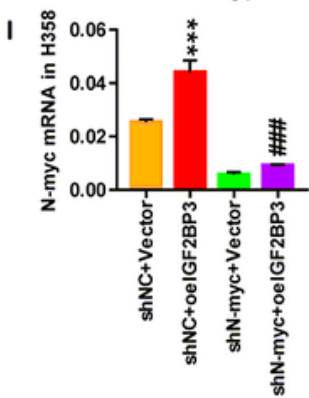

N-myc $-\ldots$
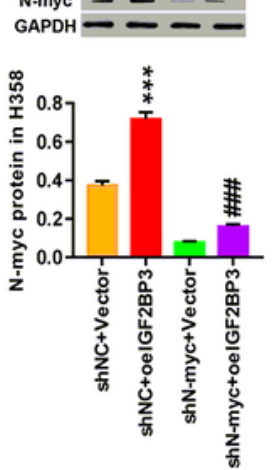

Figure 7

$\mathrm{N}$-myc is functionally important target gene of IGF2BP3 in NSCLC (A-C) CCK8 was used to detect cell proliferation in PC9 (A), H1975 (B) and H358 (C) cells in different groups. (D-F) Flow cytometry was used to detect cell cycle state in PC9 (D), H1975 (E) and H358 (F) cells with different treatment. (G-H) Q-PCR (top) and WB (bottom) were used to detect the expression of N-myc in PC9(G) and H1975 cells (H) after different treatments. (I) Q-PCR (top) and WB (bottom) were used to detect the expression of N-myc in 
H358 cells with different treatments. ${ }^{\star} p<0.05,{ }^{*} p<<0.01,{ }^{\star \star *} p<0.001$ vs. Vector+shNC or shNC+Vector; $\# p<0.05, \# \# \# p<0.001$ vs. Vector+shIGF2BP3 or shNC+oelGF2BP3.

\section{Supplementary Files}

This is a list of supplementary files associated with this preprint. Click to download.

- FigureS1.tif

- FigureS2.tif

- SupplementaryMaterial.docx

- TableS1.docx

- TableS2.xlsx

- TableS3.xIsx 